actuellement pour améliorer les traitements du lait dans les laiteries, nous pensons toujours à cette ville qui aurait fait installer une prise d'eau à côté d'une bouche d'égout et qui se préoccuperait davantage de filtrer son eau par tous les moyens que de changer sa prise d'eau de place.

Nous croyons qu'il faudrait s'occuper un peu moins de tous ees traitements mécaniques et un peu plus de la production. Car sinon nous arriverons bien un jour à fabriquer du lait synthétique, beaucoup crieront au miracle et nous annonceront le régime des. plus beaux enfants, de tous les temps. Il est vrai aussi qu'avec un peu d'adroite publicité !...

Le ramassage du lait de ferme en ferme et aux abords des chemins et des haies a fait son temps. C'est cela qu'il faut supprimer. Une seule solution, le local de coulage par village où soir et matin les producteurs apporteront leur lait. Les régions où se fabriquent les pâtes dures s'en trouvent bien, et n'adopteraient certainement. pas d'autre façon de faire. Et quelle facilité pour le contrôle.

(A suivre.)

\title{
L'APPROVISIONNEMENT EN LAIT DE LA NATION. PRODUCTION HYGIÉNIQUE ET CONTROLE
}

\author{
par BEN DAVIES
}

Directeur des laboratoires "United Dairies Limited" (Londres).

$$
\text { (Fin) }
$$

Un intéressant corollaire de cette expérience fut fourni lorsque six des douze fermiers, après leur expérience de production de lait propre, furent invités à visiter six autres producteurs et à faire la traite de leurs vaches, un après-midi, pour démontrer ainsi quelle amélioration pouvait être obtenue, sans aucune préparation spéciale, mais en se servant de leurs propres ustensiles stérilisés, qu'ils prirent avec eux. La première colonne du tableau suivant donne les.

TABLEAU E.

\begin{tabular}{|c|c|c|c|}
\hline \multirow{2}{*}{\multicolumn{2}{|c|}{ Ferme }} & Propriétaire & Visiteur \\
\hline & & $6 / 3 / 33$ & $10 / 3 / 33$ \\
\hline 1 & $\ldots \ldots \ldots \ldots$ & 3.000 .000 & 2.500 \\
\hline 2 & $\ldots \ldots \ldots \ldots$ & 3.000 .000 & 1.900 \\
\hline 3 & $\ldots \ldots \ldots \ldots$ & 25.400 & 5.500 \\
\hline 4 & $\ldots \ldots \ldots \ldots$ & 70.000 & 2.900 \\
\hline 5 & $\ldots \ldots \ldots \ldots \ldots$ & 24.500 & 900 \\
\hline 6 & $\ldots \ldots \ldots \ldots$ & 3.000 .000 & 500 \\
\hline
\end{tabular}


Dans chaque cas, la teneur bactérienne déterminée par eultures sur plaques fut :

\begin{tabular}{|c|c|c|c|}
\hline \multirow{2}{*}{\multicolumn{2}{|c|}{ Ferme }} & Propriétaire & Visiteur \\
\hline & & $6 / 3 / 33$ & $10 / 3 / 33$ \\
\hline 1 & $\ldots \ldots \ldots \ldots$ & 80.000 & 100 \\
\hline 2 & $\ldots \ldots \ldots \ldots$ & 100.000 & 200 \\
\hline 3 & $\ldots \ldots \ldots \ldots$ & 600 & 100 \\
\hline 4 & $\ldots \ldots \ldots \ldots$ & 3.900 & 100 \\
\hline 5 & $\ldots \ldots \ldots \ldots$ & 1,000 & 300 \\
\hline 6 & $\ldots \ldots \ldots \ldots$ & 200.000 & 100 \\
\hline
\end{tabular}

teneurs bactériennes normales de chacune des fournitures quatre jours avant la traite faite par les visiteurs, tandis que les résultats fournis par le travail de ces derniers est porté dans la deuxième colonne.

A la ferme $\mathrm{n}^{\circ} 2$, la traite était faite à la machine. Dans ce cas, la traite de la moitié du troupeau fut faite par le propriétaire avec sa machine ; la traite de l'autre moitié fut faite avec la machine stérilisée du visiteur, qui avait été amenée à la ferme. Les résultats furent les suivants :

\begin{tabular}{r|c|c}
\hline \hline & Propriétaire & Visiteur \\
\hline $\begin{array}{r}\text { Avant la pasteu- } \\
\text { risation ...... }\end{array}$ & 3.000 .000 & 1.900 \\
$\begin{array}{r}\text { Après la pasteu- } \\
\text { risation ...... }\end{array}$ & 31.000 & 200 \\
\hline \hline
\end{tabular}

Les méthodes de production, qui doivent être appliquées pour obtenir régulièrement un tel standard bactériologique, n'exigent pas un cours préliminaire de bactériologie élémentaire pour les producteurs de lait et leurs trayeurs. Il suffit de dicter quelques règles positives et dogmatiques dont on ne peut s'écarter, que les gens simples et laborieux, qui produisent une grande partie du lait utilisé par le pays, peuvent facilement comprendre et appliquer. Ces méthodes ne sont pas inspirées par la science, mais par les exigences de la propreté ordinaire, qu'on est en droit de demander pour la production et la préparation de toute denrée destinée à l'alimentation humaine.

Par l'observation de ces règles, la production de lait propre devient, pour ainsi dire, automatique et même inévitable. D'autre part, lorsqu'on les néglige ou qu'il y a relâchement, les épreuves de 
laboratoire proposées permettront de le découvrir bientôt et de déclasser le lait dans la catégorie inférieure, à laquelle il appartient, qui n'a droit qu'à une redevance notablement inférieure. La production de lait malpropre devrait être rendue un luxe trop coûteux. Le lait malpropre devrait être définitivement exclu de la consommation humaine. Aucun producteur, dont la fourniture n'a pas les qualités, qu'on peut exiger pour le lait en nature, ne peut prétendre récolter les bénéfices que présente la vente du lait en nature. S'il est prouvé que les épreuves de laboratoire fournissent des données correctes et que les conditions nécessaires pour obtenir la qualité bactériologique du lait exigée sont raisonnables et de réalisation pratique, alors seulement, la redevance due au producteur dépendra uniquement de la qualité même du lait, - sans que la nature des locaux, de l'outillage ou autres facteurs puissent intervenir. Si un prix réduit pour une fourniture médiocre n'a pas pour effet une amélioration immédiate de la production, cette fourniture devra être exclue du marché. Une telle action dépend, évidemment, du fait que les exigences seront raisonnables et économiques; mais, en aucune circonstance, il ne pourrait être toléré qu'un distributeur bénéficie de la vente d'un lait de qualité inférieure qu'il achète à un prix réduit.

On soutient parfois qu'on obtient une amélioration satisfaisante par le paiement de primes pour la production de lait propre, et qu'un tel système est préférable à l'application de la sanction définie, qui est proposée ici. En dehors du principe impliqué, que nous avons déjà diseuté, on peut dire qu'il n'existe que peu de preuves de la réussite réelle de ee système. Dans le cas, que nous avons déjà mentionné, où un système de prime ou de gratification, à titre d'expérience, a été en vigueur depuis plusieurs années, la redevance n'est pas seulement accordée à ceux qui obtiennent les meilleurs résultats, mais à tous ceux dont la fourniture est conforme au standard déterminé. Ce standard est assez large pour permettre que durant le mois de mars, sur 420 producteurs, la prime pût être accordée à 337 d'entre eux ; mais au mois d'août, seulement cinq sur 420 fournisseurs, eurent droit à une prime. C'est un bien mauvais système, qui fait faillite à la saison même de l'année où il devrait fournir des résultats, et pour laquelle il a surtout été élaboré. Le paiement d'une prime peut devenir un moyen efficace pour détourner des fournitures d'un acheteur vers un autre ; mais, reconnaître l'efficience du procédé pour l'amélioration de l'approvisionnement de lait en général, e'est là donner à celui-ci qualité que malheureusement l'expérience n'a pas toujours reconnue.

Avant d'abandonner la question de la propreté de l'approvisionnement en lait, il importe de parler de la réfrigération du lait. Lorsque 
la faculté de conservation du lait est en cause, on ne discute pas généralement d'autres questions, que celles qui se rapportent à la réfrigération : dimensions du réfrigérant, importance et température de la fourniture d'eau. En présence des faits exposés dans ce mémoire, il sera difficile de faire revivre la fiction, qui si longtemps servit "d'argument" contre la pasteurisation, e'est-à-dire de prétendre encore qu'elle facilite l'écoulement dans le commerce de lait malpropre. Bien que cet argument n'ait jamais été lancé contre la réfrigération, il est certain que l'utilisation de lait de qualité médiocre dépend, pour ainsi dire, complètement de l'efficacité de sa réfrigération. Devant l'enseignement des années écoulées, il peut paraître téméraire d'affirmer que la vente de lait propre ne dépend pas de l'efficacité de la réfrigération, et que, fréquemment, sa qualité bactérienne n'est apparemment pas altérée, même lorsque la réfrigération est retardée de plusieurs heures après la traite. La démonstration en est fournie par deux séries d'expériences concernant deux échantillons de lait, dont la traite avait été faite à la machine. Pour le premier, la machine avait été bien lavée, mais n'avait pas été stérilisée. Pour le deuxième, la machine avait été soigneusement lavée et stérilisée, et la nécessité d'une telle stérilisation ne pourrait être démontrée par des faits plus intéressants et plus probants. Le premier exemple, tableau F, est du lait produit par la traite du matin. Un pot de ce lait ne fut pas à dessein réfrigéré, et, lors de son arrivée à la station de réception, il fut laissé sur la plate-forme d'arrivée ; des échantillons fưrent prélevés aux intervalles indiqués, et furent analysés. Tout indique que le lait était abondamment infecté d'organismes thermorésistants par les ustensiles non stérilisés.

TABLEAU F.

LAIT NON RÉFRIGÉRÉ (MARS, 1933).

Machine à traire bien lavée mais pas stérilisée.

\begin{tabular}{|c|c|c|c|}
\hline \multirow{2}{*}{$\begin{array}{c}\text { T'emps écoulé depuis } \\
\text { la traite }\end{array}$} & \multirow[b]{2}{*}{ Tenipérature } & \multicolumn{2}{|c|}{ Bactéries par centimètre cube } \\
\hline & & - Lait cru & $\begin{array}{c}\text { Pasteurisé } \\
\text { au laboratoire }\end{array}$ \\
\hline 2 heures ........... & $78^{\circ} \mathrm{F} \cdot\left(25^{\circ} 6 \mathrm{C}.\right)$ & 330.000 & 330.000 \\
\hline 4 heures $30 \ldots \ldots \ldots$. & $73^{\circ} \mathrm{F} \cdot\left(22^{\circ} 8 \mathrm{C}.\right)$ & 710.000 & 1.050 .000 \\
\hline 6 heures $30 \ldots$. & $68^{\circ} \mathrm{F} \cdot\left(20^{\circ} 0 \mathrm{C}.\right)$ & 680.000 & 1. 500.000 \\
\hline 7 heures & $65^{\circ} \mathrm{F} .\left(18^{\circ} 3 \mathrm{C}.\right)$ & 760.000 & $3.150,000$ \\
\hline 8 heures $30 \ldots$ & $61^{\circ} \mathrm{F} \cdot\left(16^{\circ} 1 \mathrm{C}.\right)$ & 1. 190.000 & 4.410 .000 \\
\hline
\end{tabular}

Le deuxième exemple, tableau G, est encore plus caractéristique. Le lait avait été produit en été (le soir du 13 juin 1933) et 
les constatations furent faites le jour suivant (14 juin) de 17 à 24 heures après la traite. Le pot de lait, non réfrigéré, fut laissé à la laiterie, fut livré à la station de réception le matin suivant et des échantillons furent prélevés aux intervalles mentionnés. Dans ce cas, toutes les parties de la machine à traire avaient été efficacement stérilisées. Le temps était chaud, mais malheureusement les températures ne furent pas relevées.

TABLEAU G.

LAIT NON RÉFRIGÉRÉ (JUIN, 1933).

Ustensiles stérilisés, traite mécanique.

\begin{tabular}{|c|c|c|c|}
\hline \multirow{2}{*}{$\begin{array}{c}\text { Temps écoulé depuis } \\
\text { la traite }\end{array}$} & \multirow{2}{*}{ Températures } & \multicolumn{2}{|c|}{ Bactéries par centinètre cube } \\
\hline & & Lait cru & $\begin{array}{c}\text { Pasteurisé } \\
\text { nu laboratoire }\end{array}$ \\
\hline 17 heures . . . . . . . . & (non prises) & 26.000 & 20 \\
\hline 19 heures ............ & & 12.300 & 50 \\
\hline 22 heures $30 \ldots \ldots \ldots$ & & 29.000 & 30 \\
\hline 24 heures . . . . . . . . . & & 36.000 & 40 \\
\hline
\end{tabular}

De tels résultats sont remarquables, spécialement lorsqu'on sait qu'à l'œil on ne pouvait noter que peu de différence dans la propreté de l'outillage pour les deux cas. Dans le premier cas, des bactéries résistantes, thermophiles, de la machine à traire, infectèrent abondamment le lait et s'y multiplièrent au cours de la pasteurisation. La deuxième expérience démontra que dans du lait, produit avec un outillage propre, il n'y eut pas d'augmentation de la teneur bactérienne à la suite de l'omission de la réfrigération. A moins que le réfrigérateur soit bien stérilisé avant l'utilisation, il partage avec les filtres la responsabilité de faire plus de mal que de bien.

Une démonstration plus évidente encore est fournie par le tableau $\mathrm{H}$, qui donne des indications concernant d'autres échantillons de lait, qui n'avaient pas été réfrigérés. Dans ce cas, la traite avait été faite a la main, et, même lorsque non réfrigéré le lait fut laissé à des températures de $80^{\circ}$ à $70^{\circ} \mathrm{F}$. $\left(26^{\circ} 7\right.$ à $21^{\circ} 1$ C.), il n'y eut pas de modification apparente sur sa qualité bactérienne. Les exemples donnés ne doivent pas être considérés comme des critiques de la réfrigération, mais comme une démonstration de l'importance vitale d'une production propre. Car le froid ne corrige pas, il "conserve", il "stabilise ».

On ne pourrait trouver d'exemple plus frappant de l'influence de méthodes propres de production que dans l'exemple suivant que 
TABLEAU H.

LAIT NON REFRIGÉRÉ (OUTILLAGE STÉRILISÉ).

Date : 11 mai 1933, traite à la main.

\begin{tabular}{|c|c|c|c|}
\hline \multirow{2}{*}{$\begin{array}{c}\text { Temps écoulé depuis } \\
\text { la traite }\end{array}$} & \multirow[b]{2}{*}{ Températures } & \multicolumn{2}{|c|}{ Bactéries par centimècre cube } \\
\hline & & Lait eru & $\begin{array}{c}\text { Pasteurisé } \\
\text { au laboratoire }\end{array}$ \\
\hline 2 heures ..... & $74^{\circ} \mathrm{F} .\left(23^{\circ} 3 \mathrm{C}.\right)$ & 2.900 & 1.100 \\
\hline 4 heures & $71^{\circ} \mathrm{F} \cdot\left(21^{\circ} 7 \mathrm{C}.\right)$ & 4.400 & plaque nette \\
\hline 6 heures & $70^{\circ} \mathrm{F} .\left(21^{\circ} 1 \mathrm{C}.\right)$ & 3.900 & plaque nette \\
\hline 8 heures . . . . . . . . . & $70^{\circ} \mathrm{F} \cdot\left(21^{\circ} 1 \mathrm{C}.\right)$ & 4.600 & plaque nette \\
\hline 10 heures ........... & $70^{\circ} \mathrm{F} .\left(21^{\circ} 1 \mathrm{C}.\right)$ & 19.900 & plaque nette \\
\hline
\end{tabular}

Date : 16 mai 1933. Le même producteur.

\begin{tabular}{|c|c|c|c|}
\hline \multirow[b]{2}{*}{$\begin{array}{l}\text { Temps écoulé depuis } \\
\text { la traite }\end{array}$} & \multirow[b]{2}{*}{ Températures } & \multicolumn{2}{|c|}{ Bactéries par centimètre ctibe } \\
\hline & & Lait eru & $\begin{array}{l}\text { Pasteurisé } \\
\text { au laboratoire }\end{array}$ \\
\hline 2 heures & $80^{\circ} \mathrm{F} .\left(26^{\circ} 7 \mathrm{C}.\right)$ & 2.800 & 2.460 \\
\hline 4 heures & $76^{\circ}$ F. $\left(24^{\circ} 4\right.$ C. $)$ & 3.900 & 2.450 \\
\hline 6 heures ........... & $72^{\circ} \mathrm{F} .\left(22^{\circ} 2 \mathrm{C}.\right)$ & 4.300 & 40 \\
\hline 8 heures . . . . . . . . . . & $70^{\circ} \mathrm{F} \cdot\left(21^{\circ} 1 \mathrm{C}.\right)$ & 3.800 & 20 \\
\hline 10 heures & $69^{\circ} \mathrm{F} .\left(20^{\circ} 0 \mathrm{C}.\right)$ & 9.900 & plaque nette \\
\hline
\end{tabular}

nous avons connu et que nous rapportons. Du lait fut produit en Afrique, seulement à quatre-vingts milles au Nord de l'Equateur, par de la main-d'œuvre indigène sous la surveillance attentive d'un fermier anglais et de sa femme. Il n'y avait ni glace, ni eau de réfrigération, mais grâce au soin apporté dans le traitement à l'eau bouillante de tous les ustensiles et à la propreté méticuleuse de toutes les manipulations, malgré les températures tropicales, le lait résista à un voyage de cent milles vers la côte, puis fut embarqué et utilisé dans de bonnes conditions au cours du voyage vers l'Angleterre.

Dans certaines régions du pays, la traite est régulièrement faite dans les champs, à des distances considérables des fermes, où seulement des dispositions pour la réfrigération peuvent être prises. Dans bien des cas, on ne dispose pas du nécessaire pour un lavage adéquat des ustensiles. Lors d'usage de machines à traire portatives, les mêmes difficultés pour la réfrigération doivent se présenter, parce qu'il serait pratiquement impossible de transporter de grandes quantités d'eau froide pour la réfrigération du lait. Les faits qui ont été exposés démontrent que, lorsque les ustensiles pour la traite sont 
propres et ont été stérilisés, la qualité du lait ne sera pas influencée par un retard dans la réfrigération, mais que, de toute façon, la nécessité d'un outillage propre et stérilisé est impérative et péremptoire.

\section{LA SECURITÉ DE LA FOURNITURE DE LAIT Nécessité de la pasteurisation.}

Nous avons suffisamment insisté sur les moyens d'assurer une propreté convenable de la fourniture de lait, mais, pour ce qui concerne sa salubrité - l'absence dans la fourniture de lait de bactéries pathogènes, - il faut que chaque producteur prenne attentivement en considération quelques faits, qui s'imposent chaque jour à l'attention de l'administrateur, du médecin, du savant, qui ont le devoir de les étudier. Ces faits sont de telle nature que celui qui ne veut pas les voir ou en entendre parler, ou qui cherche à atténuer ce qu'elles ont de menaçant sous le souffle chaud d'un optimisme frivole, n'est pas un ami du producteur, ni de l'industrie. En vue d'obtenir des informations directes et dignes de confiance sur la situation actuelle, le "Research Advisory Committee" (Commission consultative de recherches) des "United Dairies" a entrepris une enquête eoncernant les caractéristiques des fournitures ordinaires de lait avant et après la pasteurisation et des fournitures de lait certifié et de lait "Grade A Tuberculin Tested " (catégorie A, provenant de vaches tuberculinées), qui sont produites avec licence du Ministère de l'Hygiène. Les recherches sont encore en cours, 43 enquêtes différentes de chaque catégorie ont été achevées, et pour donner une idée de la façon dont elles furent faites, nous dirons que dans certains eas, le lait de chacun des trayons de toutes les vaches d'un troupeau fut examiné séparément.

Les résultats de ces enquêtes permettent de déclarer qu'il est impossible, n'importe quel jour, de rassembler une quantité importante de lait dans n'importe quelle région grande productrice de lait de ce pays, dans laquelle on ne trouvera pas de bacilles tuberculeux vivants. Hormis là, où il est assaini par une pasteurisation efficace, partout ce lait infecté est livré au public pour être consommé dans cette condition. Dans chacun des 43 cas, le bacille tuberculeux était présent dans le lait ordinaire avant la pasteurisation, il en était absent après la pasteurisation.

Il faut rappeler que ces fournitures ordinaires constituent l'approvisionnement de $99,5 \%$ de la population, tandis qu'actuellement toute l'attention est concentrée sur l'approvisionnement du $0,5 \%$ qui reste, représenté par la fourniture des "super-troupeaux ", qui dépendent de l'Autorité catégorisatrice et distributrice de licences du Ministère de l'Hygiène. Dans une série d'échantillons de 43 
troupeaux producteurs de lait certifié ou de lait "Grade A Tuberculin Tested" (catégorie A de vaches tuberculinées), furent trouvés un échantillon nettement tubereuleux et un échantillon douteux. Le pourcentage n'est pas élevé, mais, parce que ce lait provient de troupeaux régulièrement tuberculinés et est produit avec licence du Ministère de l'Hygiène, certains médecins peu perspicaces et certains parents confiants le considèrent garanti sans danger, et e'est à l'état eru, qu'il est donné à des enfants en bas âge.

De cette même série de 43 échantillons provenant de troupeaux à production garantie par la licence, qui nécessairement sont examinés au moins tous les six mois par des médecins-vétérinaires, pas moins de 33 échantillons sur $43(76 \%)$ contenaient des streptocoques hémolytiques de la mammite, et dans 27 échantillons sur $43(62 \%)$ fut constatée la présence de Brucella abortus : deux bactéries qui peuvent provoquer des maladies graves chez l'homme. Dans quelle autre industrie tolérerait-on, un seul jour, sans prendre des mesures répressives, l'existence de tels dangers potentiels ? De droit, Sayage a pu dire dans son ouvrage (La Prévention de la tuberculose humaine d'origine bovine) : "Le fait reste qu'il (le possesseur de la vache) est, pour autant qu'il m'est connu, le seul commerçant à qui il est permis de vendre un aliment empoisonné (du lait infecté par le bacille tuberculeux) sans être traduit en justice. Le boucher qui a vendu de la viande tuberculeuse est condamnable. Le lait tubereuleux est bien plus dangereux que la viande tuberculeuse. $)$

Cette situation constitue une menace permanente pour la santé, le bien-être, la vie même, pour toutes les classes de la société - les riches aussi bien que les pauvres. Le traitement d'un seul cas de tubereulose osseuse ou articulaire, qui peut être dû à l'infection par le lait tubereuleux, coûtera soit à l'individu lui-même ou à la communauté de nombreuses centaines de livres sterling, et à la fin, lorsque toutes les ressources de la science sont épuisées, le mieux qu'on puisse espérer n'est pas la réparation des ravages provoqués, mais seulement un arrêt de l'affection. A moins de considérer comme fantaisistes et même mensongères les affirmations des savants, qui déclarent que le bacille tuberculeux bovin est transmissible à l'homme, et de penser que le rapport établi à la suite de recherches longues et minutieuses, entre le bacille de l'avortement épizootique et l'agent de la fièvre ondulante de l'homme est également une fiction scientifique, que les liens qu'on déclare exister entre certaines affections de la mamelle de la vache et certaines maladies épidémiques alarmantes et souvent fatales de l'homme n'existent que dans l'imagination (une de ces épidémies se produisit, il n'y a pas longtemps encore, en Angleterre ; il y eut un millier de malades et plus de 
quarante décès), à moins qu'on ne considère toutes ces déclarations, bien qu'elles soient appuyées par des autorités scientifiques indiscutables, comme mensongères, il est difficile de comprendre et d'admettre que l'on se laisse rassurer par de vagues généralités dans le genre de "sécurité raisonnable " ou que l'on se contente de l'éventuelle et future extension de l'inspection clinique vétérinaire des troupeaux.

Même si on devait admettre que ces dangers aient été exagérés ou surestimés, on ne pourrait nier qu'ils existent, que leur gravité n'a pas diminué jusqu'ici et qu'on ne parviendra à les maîtriser que dans un avenir éloigné, que ne connaîtra pas la génération actuelle. Comme la connaissance de faits semblables à ceux qui ont été exposés tend à se généraliser de plus en plus, comment peut-on espérer que la confiance dans la salubrité, la propreté, la pureté de l'approvisionnement en lait, dont dépend à un tel degré la prospérité de l'industrie laitière, puisse augmenter ?

Apparemment on s'est rendu compte dans les milieux gouvernementaux, qu'aucune des propositions de la "Milk Re-organization Commission " (Commission de réorganisation du lait) n'aborde le point vital d'un approvisionnement en lait salubre, sinon le "Economic Advisory Council ”. (Conseil de recherches économiques) n'aurait pas été chargé de faire un rapport à ce sujet. Bien que nous n'ayons pas l'intention de critiquer le rapport de la "Commission ", nous émettons respectueusement l'avis qu'elle' a été mal conseillée lorsqu'elle a admis et fait sienne la proposition futile du " producteur accrédité "). Il est incompréhensible que des producteurs, dont les fournitures ne sont pas plus libres de dangers potentiels que ceux de n'importe quel autre producteur, soient " accrédités " et bénéficient de primes spéciales.

En dehors de la sauvegarde positive contre tous les dangers du lait que donne la pasteurisation, on n'a pas pù proposer d'autre mesure possédant une efficacité réelle, pour garantir la "sécurité » du lait. Si nous consultons les registres scientifiques de l'organisation de recherches des "United Dairies", nous constatons que de tous les troupeaux examinés, il n'y en eut qu'un - et seulement un - dont le lait fournit des données négatives aux essais destinés à déceler l'existence de la tuberculose, de l'avortement épizootique et de la mammite. Est-ce que les adversaires de la pasteurisation penseraient que plutôt que de conférer la protection de la pasteurisation à la fourniture de lait, il faudrait envisager de décimer les troupeaux laitiers du pays, dans le but d'obtenir l'éradication de ces affections, ou bien que les consommateurs de lait doivent continuer à risquer les chances d'infection et de maladie? Il faudrait, dans de telles 
circonstances, faire montre d'un cynisme rare, pour oser eneore préconiser " un quart (1 litre 14) de lait par jour pour chaque enfant ".

N'est-il pas temps que le gros de l'industrie laitière montre un peu de sagesse, lorsque celle-ci semble avoir abandonné certains de ses chefs?

La pasteurisation est et restera probablement toujours un facteur indispensable de la garantie de la salubrité de l'approvisionnement en lait ; cette vérité est démontrée de nouveau chaque jour, tant par les recherches scientifiques que par les données de la clinique. Il n'est pour ainsi dire pas nécessaire d'apporter de nouvelles preuves d'un fait, déjà amplement prouvé et généralement accepté. La sincérité de la majorité des producteurs et des vendeurs de lait, adversaires de la pasteurisation, sera durement mise à l'épreuve lorsqu'on les rendra définitivement responsables de la présence, dans leurs fournitures, de germes microbiens pouvant provoquer des maladies ehez le consommateur. Le fait qu'une source d'infection, qui pourrait facilement être supprimée, persiste et continue d'être une menace-pour la santé de la fraction de la population à laquelle le lait est le plus nécessaire et qui est la plus susceptible d'infection, est un obstacle au progrès de l'industrie laitière.

\section{LA SALUBRITÉ DE L'APPROVISIONBEMENT EN LAIT}

Nous avons essayé de définir les conditions dans lesquelles on peut procurer positivement et rapidement une fourniture de lait propre et salubre à la grande majorité de la population. Il est possible d'obtenir ce résultat d'une importance primordiale, mais il est également urgent de prendre toutes les mesures possibles pour améliorer l'état de santé général des troupeaux laitiers du pays - ce qui est d'une importance inestimable au point de vue économique. La réduction de l'incidence des maladies du cheptel exige des plans, qui ne doivent pas être élaborés pour quelques années, mais pour les générations futures, et pour la solution des différents problèmes il faudra probablement recourir à toutes les ressources que peuvent offrir l'hygiène, l'administration et la finance.

Nous avons déjà fait allusion aux aspects complexes du problème. Comme celles de l'être humain, les maladies du bétail sont dues à l'infection communiquée généralement d'un animal à l'autre ; mais assez fréquemment elle est transmise à l'animal par l'homme qui le soigne, et les mesures prises pour combattre de telles infections doivent être appropriées à chaque cas. Ici la question doit se poser de savoir par quelles considérations pratiques et économiques il faudra se laisser guider pour étendre l'influence des facteurs d'éducation, d'assainissement et d'isolement des individus atteints. Les maladies prédominantes du bétail présentent une incidence prati- 
quement universelle, et s'il était possible de peupler chaque ferme d'un nouveau troupeau de vaches indemnes de toute maladie, il est probable qu'après une année, s'il n'y avait pas eu amélioration des conditions de logement et modification des méthodes, la situation redeviendrait semblable à la précédente.

Nous ne pouvons perdre de vue que la majorité des étables et des abris pour le bétail constitue un héritage d'une période à laquelle on avait à peine quelques notions d'hygiène et au cours de laquelle le travail était mal payé au point qu'il était à peine nécessaire d'en étudier le rendement. Dans de tels locaux, mal construits et mal aménagés, le bétail est assemblé, enfermé pendant des mois, mangeant à la même mangeoire, buvant au même abreuvoir, respirant et respirant à nouveau le même air. Qui, n'ayant même que des notions élémentaires concernant l'infection et la contagion, espérerait maintenir un animal en bonne santé, dans un tel milieu. La condition initiale et indispensable à n'importe quel progrès est la compréhension ; vouloir combattre la maladie, sans avoir éliminé les causes d'infection, constitue une absurdité.

Si l'on veut obtenir des troupeaux sains, des fournitures de lait satisfaisantes, si on veut arriver à une production économique, une des premières questions qui doit être prise en considération est la possibilité de remplacer, dans des conditions pas trop onéreuses, les antres obscurs, qui servent actuellement au logement du bétail, par des locaux hygiéniques permettant le maintien de la propreté et de la santé des troupeaux. Il a déjà été démontré qu'à peu de frais il est possible d'améliorer, jusqu'à un degré qui peut surprendre, la propreté, la pureté des fournitures de lait. Dans le cas où, à cause de la construction de nouveaux locaux, des dépenses importantes sont inévitables, l'aide du Gouvernement devrait dans une certaine mesure être obtenue, si on voulait rendre possible une amélioration générale. Une telle aide devrait être accordée sur une base strictement économique, et le plus avantageux serait, peut-être, de garantir les intérêts de prêts accordés, à cette fin, par des particuliers et des organismes financiers. Il est à penser que la dépense serait immédiatement profitable au producteur, à cause des économies de travail qu'elle entraînerait, tandis que les intérêts financiers considérables des banques, investis dans les propriétés fermières et terriennes, seraient sauvegardés et consolidés.

Il n'est pas nécessaire d'aller plus loin que l'énonciation du principe. Les détails sembleraient évidents, ils comprennent une ventilation adéquate et sans courants d'air, de la lumière tant naturelle qu'artificielle en abondance, des passages devant et derrière les vaches pour l'affouragement et l'enlèvement du fumier, des bassins d'abreuvage individuels et des mangeoires individuelles, ces 
dernières à un niveau peu éloigné du sol et adéquatement séparées de celles des stalles et des vaches voisines; des stalles pourvues d'une aire imperméable et non eonductrice, des dispositifs d'attache des vaches, qui ne les forceraient pas à se coucher dans leurs excréments, des commodités appropriées, des lavabos pour le personnel, des aménagements pour le nettoyage, la stérilisation et la resserre des ustensiles, ainsi que pour la réfrigération rapide du lait dans un milieu souillé.

Dans bien des cas, il serait possible d'adapter les locaux existants, sans grandes dépenses; les détails mentionnés sont indispensables pour l'obtention des résultats satisfaisants. Une grande importance doit être attachée à l'existence d'un passage entre le mur et les animaux, non seulement pour aider à la ventilation, mais encore pour préserver les fourrages des contaminations. On a l'habitude de déposer à terre, derrière les vaches, sans faire attention à la propreté de l'aire, des bottes ou des brassées de fourrage. Le fumier étant souvent infecté par des bacilles tuberculeux, la souillure du fourrage ainsi provoquée favorise l'infection des animaux. Il suffit d'observer les vaches au pâturage pour constater que, naturellement, elles demandent une alimentation propre et sans souillures.

Si, en même temps, des dispositions étaient prises pour la conservation et l'utilisation du fumier liquide, et si l'on procédait au transport automatique et mécanique du fumier et de la litière souillée vers une resserre adéquate, les vaches ne devraient plus traverser des amas d'excréments pour arriver aux abris destinés à la traite; le travail de nettoyage préliminaire des femelles laitières, indispensable pour que la traite puisse être faite dans les conditions de propreté et d'hygiène nécessaire, serait fortement réduit. Les égards dus à un aliment destiné à l'alimentation de l'homme devraient suffire pour rendre obligatoires ces conditions désirables, et si, ensuite, l'indifférence persistait, on pourrait avoir recours à une mesure de contrainte, qui, en dernier ressort, provoquerait l'exclusion du marché des fournitures malpropres.

L'aide financière, les conseils bienveillants, la standardisation des plans des locaux, la détermination d'équipements et d'outillages spécifiés ne suffiront pas ; il faudra encore insister sur l'observation des mesures indispensables à une production de lait salubre, mesures qui, actuellement, sont encore trop souvent considérées comme des fantaisies impraticables. En même temps que la fourniture de l'équipement et de l'outillage, ainsi qu'elle est proposée ici, la construction des nouveaux locaux pourra être entreprise, n'importe où, avec la main-d'œuvre locale. On peut encore faire observer qu'une telle abondance de travail dans tous les districts ruraux, sur une base économique qui n'impliquerait pas de nouvelles levées d'impôts, 
serait d'un bien plus grand bénéfice pour la communauté que certains des projets de réduction du chômage, qui ont été proposés ; elle aura encore l'avantage de fournir du travail à une notable proportion de la main-d'œuvre non spécialisée.

Passant pour le moment sous silence la deuxième question, qui est celle des modifications désirables de l'Administration, nous abordons la troisième, qui se rapporte à la valeur et aux possibilités d'application des méthodes destinées à réduire les dangers de la tuberculose bovine.

Chez les animaux, comme chez l'homme, le plus grand ennemi de la tuberculose est la vie au grand air, tandis que l'affection trouve un terrain favorable dans la stabulation quasi permanente des laitiers. Après quarante ans de tuberculination, la fréquence de la tuberculose n'a pas diminué. Le "Tuberculosis Order» (loi sur la tuberculose) prévoit une compensation suffisante pour enterrer le cadavre d'un animal, dont la mission de propager l'affection est terminée.

Il faut chercher le secret du succès dans une modification du système des primes proposé par la «Commission ». Au lieu d'accorder ces primes pour des "producteurs accrédités ", on les accordera pour des "troupeaux accrédités". La première proposition est basée sur un principe rétrograde, qui considère la production propre comme un "extra" facultatif, l'autre sur un principe logique constructif. Les affections du bétail constituent un triste héritage du passé, pour lequel il est difficiele de blâmer la génération actuelle, mais la malpropreté de la fourniture de lait est directement attribuable à l'ignorance et à l'incurie de cette génération ; on pourra y remédier dès que la question sera abordée d'une manière rationnelle.

Des sujets réagissant à la tubereuline ne pourraient se trouver dans des troupeaux accrédités, dont toutes les unités seraient tuberculinées sous contrôle officiel, deux fois par an. Les sujets fournissant une réaction pesitive à la tuberculine, seraient marqués d'une façon indélébile et ne pourraient être maintenus dans les exploitations, en même temps qu'un troupeau accrédité, que lorsque la séquestration serait possible, et alors seulement pour une période de durée limitée, ne dépassant pas, par exemple, un an. Le troupeau serait maintenu ou augmenté par l'élevage ou par l'acquisition de sujets provenant de troupeaux accrédités. Lorsque de tels troupeaux deviendraient assez nombreux dans un district, il devrait être possible de classer celui-ci "district accrédité", dans lequel il serait défendu d'introduire des sujets fournissant une réaction positive à la tubereuline. Tant la tuberculination que la fourniture de tuberculine seraient officiellement contrôlées. Les veaux, qui naîtraient de vaches donnant une réaction positive à la tuberculine, devraient 
être isolés dès la naissance. Seulement pour le lait provenant de fermes accréditées, des dénominations spéciales seraient autorisées, et seulement à ce lait pourrait être accordée une prime pour la qualité et la salubrité.

Ce mémoire deviendrait trop long, s'il fallait encore s'étendre. Il est difficile de faire plus ici que d'indiquer les principes directeurs. Qu'il faille étendre les exigences de la classification comme "troupeau accrédité " pour obtenir l'élimination d'autres maladies, cela est une question de recherches. On ne connaît pas assez le rôle de l'homme, chargé de soigner les vaches, dans la transmission de l'infection d'une vache à l'autre, par les conséquences d'une traite malpropre, mais il est plus que probable que ce rôle est important, surtout pour la mammite. Dans tous les cas, ceci renforce la nécessité de l'aménagement ou de la création d'étables hygiéniques.

Une des dernières questions, qui doit être examinée, est celle de l'autorité qui sera chargée de l'exécution des mesures destinées à améliorer l'approvisionnement en lait. La proposition de la "Milk Re-Organization Commission ") (Commission de réorganisation du lait), d'un contrôle exercé par un Conseil composé de producteurs et de distributeurs, dont les décisions sont sujettes à appel devant une Commission composée de trois membres, pourrait convenir. Il importe pourtant de signaler qu'il a été reconnu impossible d'appliquer les preseriptions du "Milk and Dairies Order" (loi sur le lait et les laiteries) dans les campagnes, parce que les conseils sont sous le contrôle de ceux qui seraient immédiatement touchés par l'application de la loi.

Il n'est pas possible de discuter toutes les questions, que les propositions émises dans ce mémoire peuvent provoquer. Il n'est pas question de traduire d'emblée un individu en justice parce que ses fournitures sont malpropres, mais la sanction qui suivra impitoyablement sera constituée par une rémunération inférieure à celle accordée pour une production propre. Avant de faire n'importe quelle proposition, nous étions convaincus, par l'expérience, que les méthodes proposées peuvent être appliquées par les producteurs les plus humbles. La propreté devra avoir une valeur bien définie, tandis que la production de lait malpropre devra devenir une prodigalité coûteuse. Les ressources financières nécessaires au service du laboratoire de contrôle pourraient être constituées par le rapport des sanctions : l'écart de prix payé pour du lait propre et du lait malpropre ; on ne peut permettre qu'un commerçant fasse des bénéfices par l'achat, à un prix peu élevé, de lait qui n'est pas conforme au standard déterminé.

Si l'industrie de la production du lait doit prospérer, si la vente du lait en nature, réellement rémunératrice pour le producteur, 
doit, dans une certaine mesure, devenir proportionnelle à la production, cela ne peut se produire que conjointement avec le développement d'une confiance générale et complète dans la propreté et la salubrité de la fourniture de lait tout entière. A cet effet, il doit être spécifié que, dans des limites bien déterminées, non seulement la production " catégorisée " et " accréditée " doit répondre aux conditions spécialement établies, mais eneore que, partout et toujours, tout lait doit être propre et de bonne qualité, et que lors de la livraison au consommateur la salubrité de tout lait doit être absolue et non pas relative. Ces exigences auraient dû être établies depuis longtemps ; actuellement, sans aucun obstacle, sans aucun empêehement, on distribue tous les jours de grandes quantités de lait, qui ne présentent pas la moindre garantie hygiénique. Si la confiance est la condition essentielle de la prospérité, peut-il exister quelque espoir pour l'industrie, aussi longtemps que les conditions actuelles ne seront pas modifiées?

$$
*^{*} *
$$

En terminant cet exposé, nous tenons à remercier, sans distinction aucune, tous les membres du personnel des laboratoires des "United Dairies" pour le concours enthousiaste qu'ils nous ont apporté au cours des recherches qui ont précédé la publication de ce Mémoire. Ces recherches spéciales eurent une durée d'environ trois ans. Au risque de froisser leur modestie, nous tenons à mentionner l'assistance, en tout temps, de MM. E. B. Anderson, L, J. Meanwell et Leslie J. Walker.

\title{
REVUE
}

\section{LA PRODUCTION MONDIALE DE CASÉINE}

\author{
par \\ G. GENIN \\ Ingénieur E. P. C.
}

La demande mondiale de caséine, quelle que soit sa qualité, s'est développée dans de grandes proportions depuis déjà quelques mois. Il en est résulté naturellement une augmentation de la production et une élévation des prix. Ce développement de la consommation provient d'un afflux de demandes de la part de l'industrie des matières plastiques, des colles, des détrempes, du papier couché, des insecticides, de l'industrie textile et naturellement des produits alimentaires de régime et des produits médicinaux.

La production mondiale de caséine (caséine à la présure et caséine 